\title{
AN ACTIVE THREE-DIMENSIONAL MICROELECTRODE ARRAY FOR INTRACORTICAL RECORDING
}

\author{
Qing Bai, Marcus D. Gingerich, and Kensall D. Wise \\ Center for Integrated Sensors and Circuits \\ Department of Electrical Engineering and Computer Science \\ The University of Michigan, Ann Arbor, MI 48109-2122
}

\begin{abstract}
This paper reports a three-dimensional (3D) microelectrode array with on-chip circuitry for use in the central nervous system (CNS). The active 3D array is created by microassembling 2D active probes and uses right-angle gold beams as lead transfers between the probes and a mounting platform. In order to improve fabrication yields and ensure successful 3D microassemblies, new bulk micromachining and encapsulation techniques have been developed to protect the onchip circuitry from the probe release etch and from the body electrolytes. Since an external clock must be used in active 3D arrays to synchronize the readouts from several multiplexed probes, clock suppression to $10 \mathrm{ppm}$ or better must be obtained in order to resolve $50 \mu \mathrm{V}$ neural signals. The CMOS probes reported here achieve this performance and have successfully recorded single-unit neural activity in vivo.
\end{abstract}

\section{INTRODUCTION}

Improved understanding of the central nervous system and the successful realization of auditory and visual prostheses to aid the handicapped are important goals for the neurosciences, but progress in both of these areas depends strongly on the development of improved microelectrode arrays for electrical recording and stimulation at the cellular level. Various micromachining technologies [1-5] have been developed over the years to create one-, two- and three-dimensional passive arrays for acute and chronic use. Multichannel 2D active probes (containing on-chip electronics) have also been reported for recording [6,7] and stimulation [8,9] in the CNS. However, there is a continuing need to develop still higher-performance arrays suitable for accessing large neuronal populations in 3D volumes of tissue using very few external leads. Such active 3D arrays can be realized by assembling 2D probes using a highyield process reported recently [10].

The fabrication of active probes requires that a CMOS IC process be merged with the boron-etch-stop process used to define the probe shanks and electrode sites while encapsulating the entire structure to shield it from the probe release etch and from body electrolytes. Although a fabrication process for 2D active probes has been reported [7, 11], further improvements were needed to achieve high yield and meet the challenges of the 3D structure. New bulk micromachining and encapsulation techniques have been developed to ensure full compatibility between the probe structure and on-chip circuitry.

With a growing demand for multi-site recording arrays and large 3D structures, it is becoming critically important to develop active probes so that multiplexing can be used to reduce external lead counts and electronically select sites that are close to active neurons. Active $3 \mathrm{D}$ multiprobe arrays also require that an external clock be supplied to all probes in the array to permit synchronization among the various outputs. Because the neural signals of interest extend down to amplitudes well under $50 \mu \mathrm{V}$, the potential noise problems associated with multiplexing such low-level signal channels pose a significant challenge, both in designing the on-chip circuitry as well as in the external demultiplexing system. This paper demonstrates bulkmicromachined CMOS active probes with externally-clocked multiplexing that maintain low noise performance and achieve high yield.

\section{FABRICATION OF ACTIVE 3D PROBES}

CMOS active probes are fabricated using a combination of the deep boron etch-stop technology used to fabricate passive probes and a $3-\mu \mathrm{m}$ p-well CMOS process. The process has 16 masking steps and consists of three main phases: 1) definition of the probe substrate, including deep and shallow boron diffusions; 2) a self-contained CMOS process sequence; and 3) formation of the iridium electrode sites and gold bonding pads, circuit encapsulation, and release of the probe from the host substrate. Although most of this process had been successfully developed previously, providing adequate encapsulation for the on-chip circuitry and achieving high yield during the probe release etch remained important issues as this work began.

Figure 1 shows the structure of an active probe for use in a 3D microassembly. The probe substrate has mounting "wings" containing slots for alignment spacers that hold the probes parallel and orthogonal to the mounting platform; electroplated gold beams form lead transfers between the probes and this platform. The probe release etch is more challenging for $3 \mathrm{D}$ active probes than for $2 \mathrm{D}$ probes because there is no etch-stop below the circuit area and the lightly doped silicon there must be retained while the probe shanks and wings are thinned to the $15 \mu \mathrm{m}$-deep etch-stop layer. This need to preserve silicon in

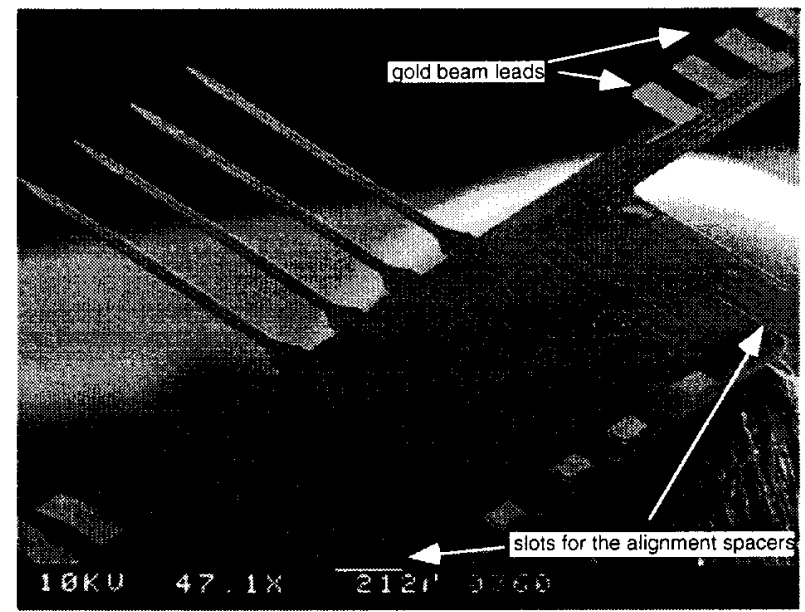

Figure 1: SEM of an active probe that has gold-plated beams for orthogonal lead transfers and slots for the alignment spacers associated with $3 D$ microassembly. 
some areas while removing it in others has made it difficult to maintain a sufficiently broad process window to ensure high yield in the past. However, satisfactory margins have now been achieved using deep RIE and dielectric corner compensation as shown in Fig. 2.

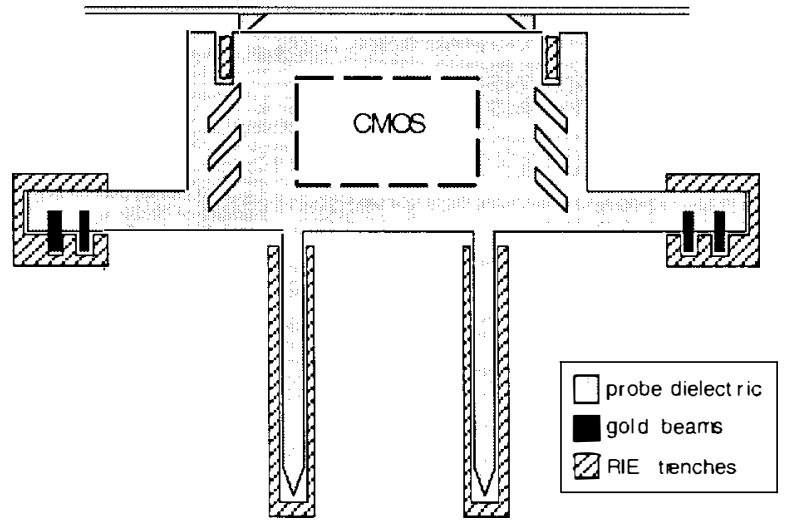

Figure 2: Diagram of an active probe that has deep RIE etched trenches around the wings and shanks to facilitate early release of those areas and corner compensation dielectric bridges to protect rear circuit area from corner undercutting during EDP.

Corner undercutting has sometimes been an important source of yield loss in active probe fabrication in spite of leaving a wide boron-doped silicon rim around the active areas. This can occur when crystallographic planes such as the $\{331\}$ are accessible to the front-side etch at outside (convex) pattern corners as shown in Fig. 3, where an unprotected corner was attacked by EDP to undercut the masking oxide. A simple way to avoid this problem is to include dielectric masking bridges at such corners (Figs. 2 and 3). preventing the \{3.31\} planes from being exposed. These corner compensation bridges provide protection until either the $\{111\}$ planes meet to undercut the bridges from the front or the front- and back-side etch planes meet each other, allowing attack of the circuit areas from below. The corner-compensation dielectric bridges break off when the probes are released from the wafer.

The mounting wings and probe shanks must be thinned to their etch-stop thickness to ensure high dimensional precision. This needs to occur from the front since the back etch would result in very thin silicon under the circuit areas and an inadequate process window. Normally, since the shanks are aligned with the $\langle 110\rangle$ directions and are only slightly tapered, any undercut from the front must work backward from the shank tips and convex corners. This process is slow and can allow the circuit areas to be attacked from the back, destroying the circuitry. To increase the process etch window, deep-etched trenches can be formed around the wings and shanks so that the front undercut etch proceeds laterally in the $\langle 110\rangle$ directions, as illustrated in Fig. 4. For 50 $\mu \mathrm{m}$-wide probe shanks, 70 $\mu \mathrm{m}$-deep slots have been etched around the probes using deep RIE, facilitating their release from the front. This leaves a thick silicon layer under the circuitry, ensuring a wide process window.

Encapsulation of the active probes is another important processing issue. Stress-compensated LPCVD oxide and nitride layers are used under the electrode interconnects, while LTO and PECVD nitride form the top passivation layers. A polymer film may be used to offer additional protection against the biological environment. In order to prevent junction spiking and aluminum hillocking during deposition of the top dielectrics, $100 \mathrm{~nm}$-thick titanium plugs are used with multiple titanium/aluminum layers for circuit metalization. This fabrication process has resulted in a high yield of $2 \mathrm{D}$ and $3 \mathrm{D}$ active probes. Figure 5 shows optical and SEM views of completed active 3D arrays.

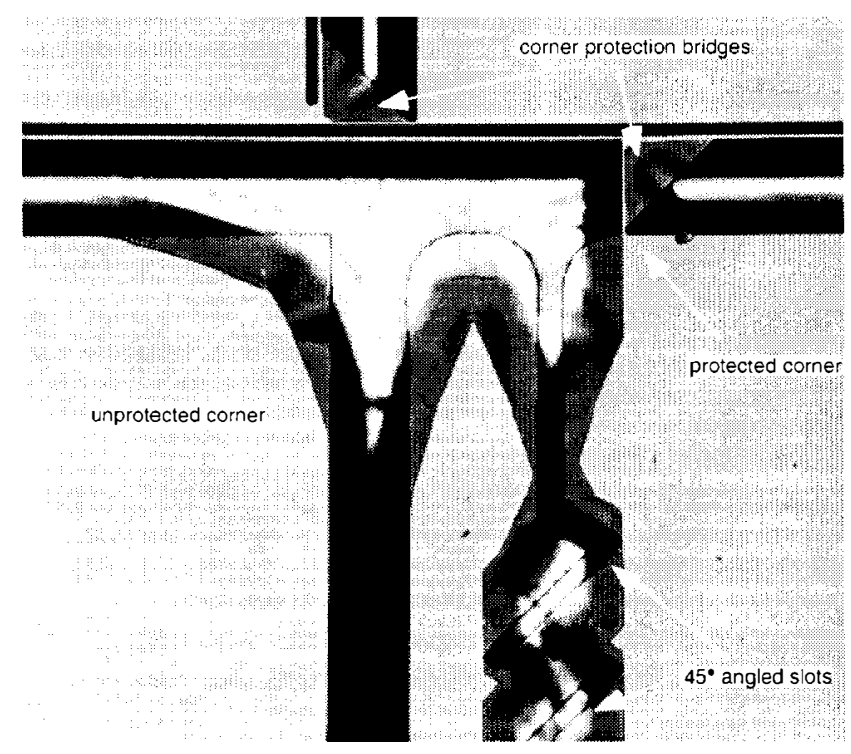

Figure 3: A testing device structure with dielectric mask after 40 min. EDP etch. It shows that circuit areas can be well-protected from corner undercutting in EDP by using corner compensation dielectric bridges. The $45^{\circ}$ angled slot openings also help to clear the wing area for the alignment spacers.

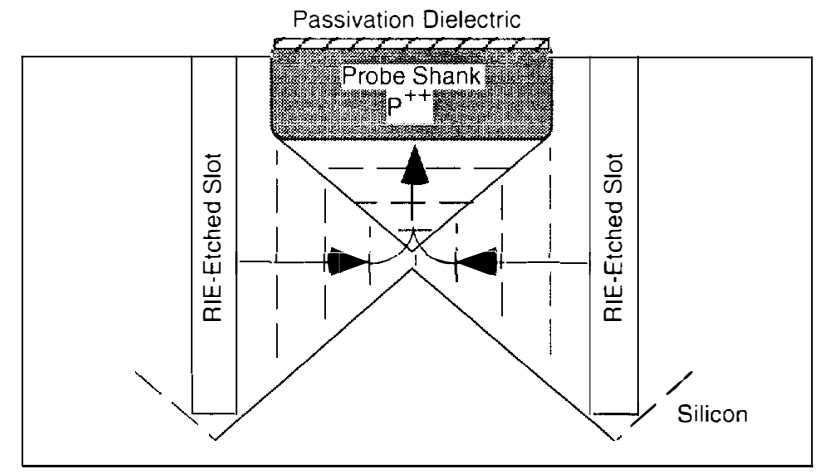

Figure 4: Cross-section of a probe shank with slots along its sides. These slots allow lateral etching to undercut the shank from the front in EDP, well before the etch front from the backside of the wafer reaches them. Thus, thick silicon can be preserved over the circuit area while the shanks and wings are released from the front.

\section{EXPERIMENTAL RESULTS}

Several active probes have been developed to test different amplifier/multiplexer designs and shielding concepts. The probes contain four shanks with multiple sites per shank and outputs that are buffered, unbuffered, multiplexed, or nonmultiplexed. The on-chip signal processing circuits on 
these probes reduce the output impedance levels, provide signal amplification, and multiplex the recorded signals to reduce the number of required external leads. However, this circuitry can also add noise to the recording channels. The measured noise from the input devices in this circuitry is typically less than 15 $\mu \mathrm{V}$-rms over the frequency range of interest $(100 \mathrm{~Hz}-10 \mathrm{kHz})$. This is less than the thermal noise from a typical recording site and does not degrade the overall signal-to-noise ratio (SNR). Recent experiments using one of the buffered probes have shown this. On this probe, a passive site was located adjacent to every buffered/active one, with a site-separation of $24 \mu \mathrm{m}$ center-to-center and a site area of $81 \mu \mathrm{m}^{2}$. The voltage buffer in this case was a simple source follower with an output impedance of $3 \mathrm{k} \Omega$ and a gain factor between 0.85 to 0.9 . Figure 6 shows in vivo recordings obtained using this probe. The on-chip buffers clearly do not degrade the overall noise performance of the recording system.
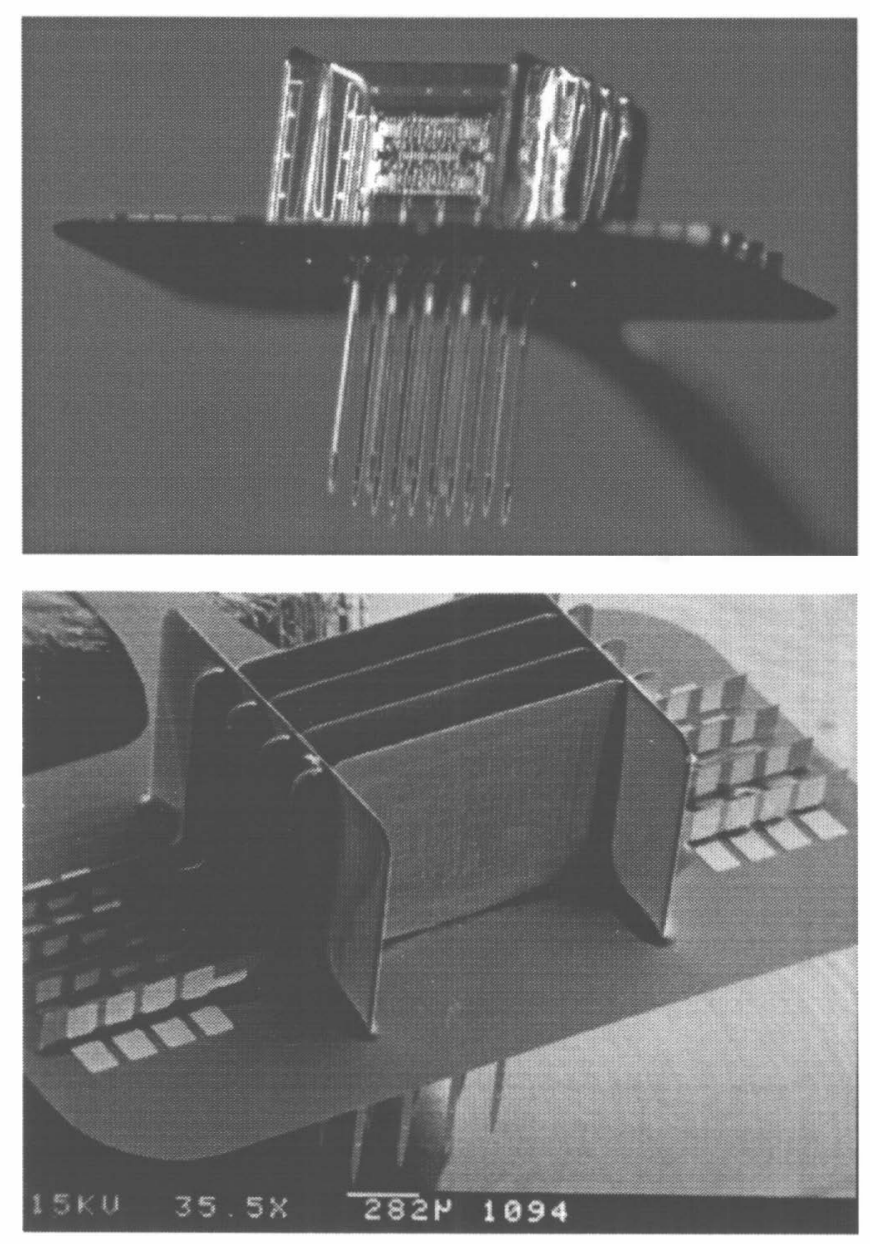

Figure 5: Optical (top) and SEM (bottom) views of the completed active $3 D$ microelectrode arrays.

In multi-probe $3 \mathrm{D}$ arrays, an off-chip clock is required to allow synchronization of the different probe outputs. However, this poses a potential noise problem and requires suppression of the $5 \mathrm{~V}$ clock signal to $10 \mathrm{ppm}$ or better. Achieving this performance, in turn, requires an understanding of all possible coupling mechanisms, both between leads and on the chip itself. Figure 7 shows the system diagram for one simple multiplexed probe and its external demultiplexing circuitry.
This probe, the simplest design of four multiplexed probes that have been developed, consists of voltage buffers, a two-bit counter, and CMOS switches. The probe was carefully laid out to minimize noise coupling. The external demultiplexing system contains three stages: a first amplification stage, a demultiplexing stage, and a second amplification/filtering stage. A $200 \mathrm{kHz}$ non-symmetrical (20\% duty cycle) clock is used with a $2 \mu$ s delay and a $2 \mu$ s sampling duration to avoid switching noise. The demultiplexing system itself has a total gain of 5000 and an equivalent input noise of less than $5 \mu \mathrm{V}$. Neural signals recorded with one of these probes are shown in Fig. 8. The noise level of the recovered demultiplexed signals is low but still higher than that of the same nonmultiplexed (clock-off) signals (about $40 \mu \mathrm{V}$ compared with $20 \mu \mathrm{V}$, respectively). The main source of this clock noise is coupling between the external clock lead and the multiplexed $10 \mathrm{k} \Omega$ dataoutput line. For closed-loop buffer designs having output impedances of $1 \mathrm{k} \Omega$ or less, the clock noise is expected to be significantly reduced $(<15 \mu \mathrm{V})$. Coupling on the probe substrate itself is very small since the substrate forms a ground plane below the interconnect lines and the extracellular fluid forms a ground plane above them in most recording situations.
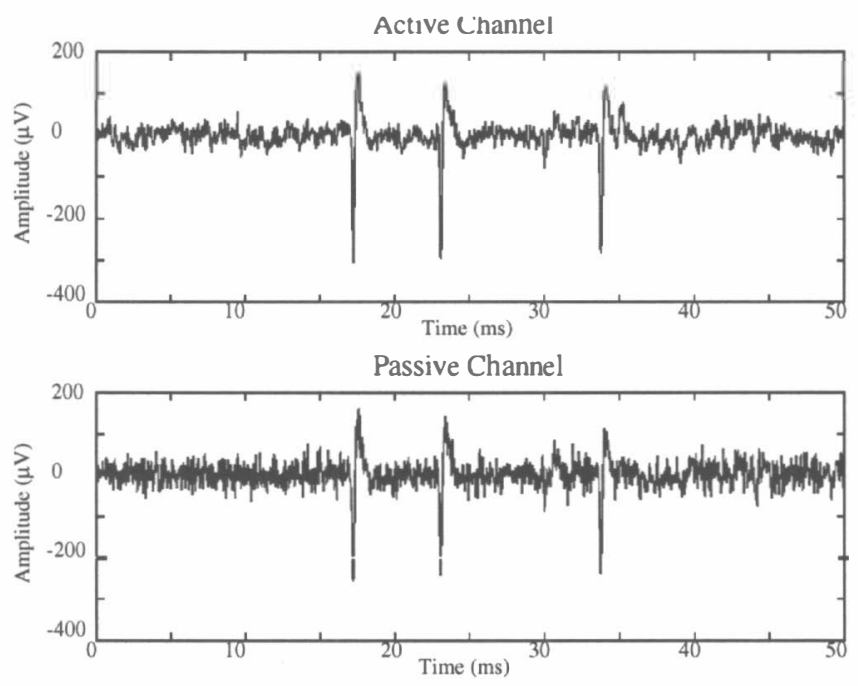

Figure 6: Spontaneous neural activity recorded through a bufferedlactive channel (above) and an unbuffered/passive channel (below), which are only $24 \mu \mathrm{m}$ apart.

\section{CONCLUSIONS}

An active $3 \mathrm{D}$ microelectrode recording array has been fabricated and tested. It was created by microassembling $2 \mathrm{D}$ active silicon probes using a right-angle beam-lead-transfer technique. A process that combines deep RIE and dielectric corner compensation has been developed to protect the on-chip circuitry from undercutting during the probe-release etch. Single-unit neural responses have been successfully recorded in vivo using these probes with an off-chip demultiplexing system, Even the simplest of these probes has exhibited clock suppression to better than $10 \mathrm{ppm}$, and probes having output resistances less than $1 \mathrm{k} \Omega$ are expected to show noise levels well below the thermal noise floor of the recording sites themselves. The test results with these active probes are forming the basis for a much larger 1024-site 3D array now in development. 


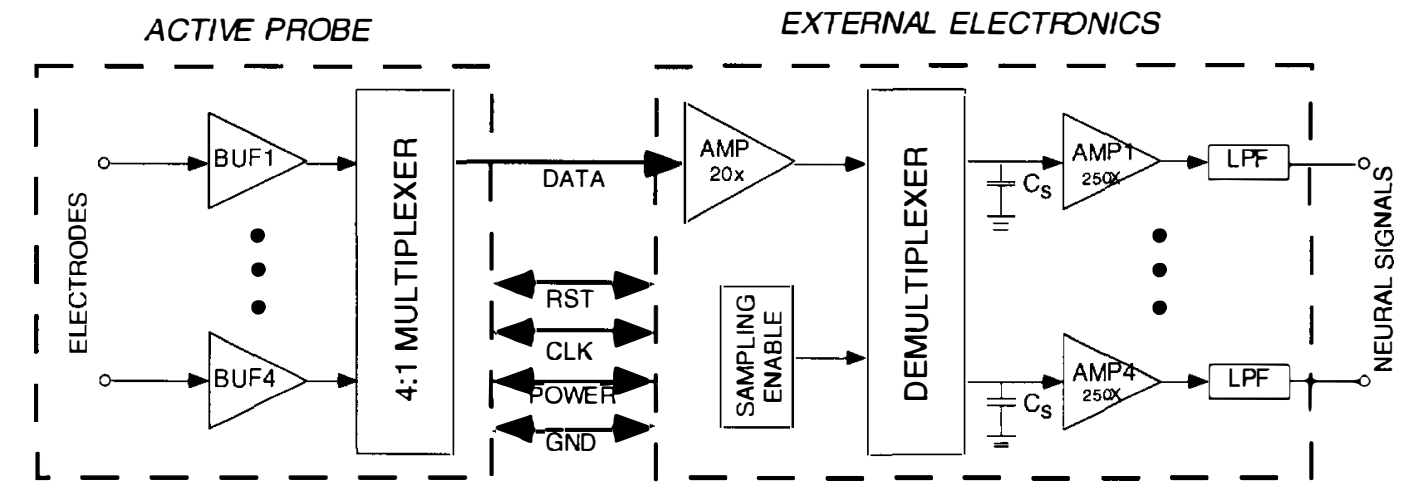

Figure 7: Block diagram of the overall on-chip multiplexing and off-chip demultiplexing system.
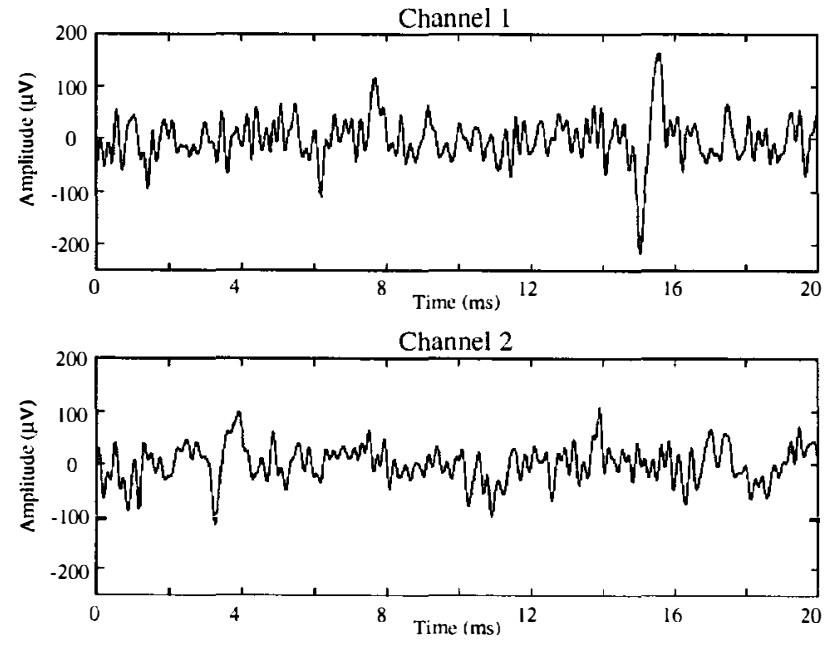

Figure 8:) Simultaneous neural signals recorded in guinea pig auditory cortex by the multiplexer-demultiplexer active probe system. The responses are driven by white noise bursts, and the two channels are $200 \mu \mathrm{m}$ apart.

\section{ACKNOWLEDGMENTS}

The authors would like to thank Drs. F. T. Hambrecht and W. Heetderks of the Neural Prosthesis Program, National Institute of Neurological Disorders and Stroke, for their encouragement and support of this work. The assistance of $\mathrm{Mr}$. J. Wiler and Mrs. J. Hetke in the animal testing were also very much appreciated.

\section{REFERENCES}

1. K. Najafi, K. D. Wise, and T. Mochizuki, "A High-Yield ICCompatible Multichannel Recording Array," IEEE Trans. Electron Devices, 32, pp. 1206-1211, July 1985.

2. J. F. Hetke, J. L. Lund, K. Najafi, K. D. Wise, and D. J. Anderson, "Silicon Ribbon Cables for Chronically-Implantable Microelectrode Arrays," IEEE Trans. Biomed. Engr., 4l, pp. 314-321, April 1994.
3. A. C. Hoogerwerf and K. D. Wise, "A Three-Dimensional Microelectrode Array for Chronic Neural Recording," IEEE Trans. Biomed. Engr., 41, pp. 1136-1146, December 1994.

4. P. K. Campbell, K. E. Jones, R. J. Huber, K. W. Horch, and R. A. Normann, "A Silicon-Based Three-Dimensional Neural Interface: Manufacturing Processes for an Intracortical Electrode Array," IEEE Trans. Biomed. Engr., 38, pp. 758-767, August 1991.

5. D. Kewley, M. Hills, D. Borkholder, I. Opris, N. Maluf, C. Storment, J. Bower, and G. Kovacs, "Plasma-Etched Neural Probes," Sensors and Actuators A, 58, pp. 27-35, 1997.

6. K. Najafi and K. D. Wise, "An Implantable Multielectrode Recording Array with On-Chip Signal Processing," IEEE J. Solid-State Circuits, 2I, pp. 1035-1044, December 1986.

7. J. Ji and K. D. Wise, "An Implantable CMOS Circuit Interface for Multiplexed Microelectrode Recording Arrays," IEEE J. Solid-State Circuits, 27, pp. 433-443, March 1992.

8. S. J. Tanghe and K. D. Wise, "A 16-Channel CMOS Neural Stimulating Array," IEEE Journal of Solid-State Circuits, 27, pp. 1819-1825, December 1992.

9. C. Kim and K. D. Wise, "A 64-Site Multishank CMOS LowProfile Neural Stimulating Probe," IEEE J. Solid-State Circuits, 31, pp. 1230-1238, September 1996.

10. Q. Bai and K. D. Wise, "A High-Yield Process for ThreeDimensional Microelectrode Arrays," Digest Solid-State Sensor and Actuator Workshop, Hilton Head, S.C., pp. 262-265, June 1996.

11. J. L. Lund and K. D. Wise, "Chip-Level Encapsulation of Implantable CMOS Microelectrode Arrays," Digest Solid-State Sensor and Actuator Workshop, Hilton Head, S.C., pp. 29-32, June 1994. 